\title{
Journal of

\section{Facile synthesis of the DD3R zeolite: performance in the adsorptive separation of buta-1,3-diene and but-2-ene isomers}

\author{
Canan Gücüyener, ${ }^{* a}$ Johan van den Bergh, ${ }^{a}$ Alberto Martinez Joaristi, ${ }^{a}$ Pieter C. M. M. Magusin, ${ }^{b}$ \\ Emiel J. M. Hensen, ${ }^{b}$ Jorge Gascon ${ }^{* a}$ and Freek Kapteijn ${ }^{a}$
}

Received 1st August 2011, Accepted 19th September 2011

DOI: $10.1039 / c 1 j m 13671 b$

\begin{abstract}
Small pore size and hydrophobic nature of DD3R make this material a unique zeolite with high potential in industrial separation applications. However, the reproducible rapid synthesis of this zeolite is still a problem. In this work, a thorough assessment of different synthetic methods revealed that synthesis reproducibility relies on two main pillars: the use of properly cleaned autoclave liners and the synthesis composition. High quality DD3R crystals are obtained when $\mathrm{KOH}$ is used as a cleaning agent, eliminating memory effects, and when KF is used in the synthesis as a mineralizing agent. The effect of fluoride addition is investigated by use of several characterization techniques $\left({ }^{13} \mathrm{C},{ }^{19} \mathrm{~F}\right.$ and ${ }^{29} \mathrm{Si}$ MAS-NMR and (2D) ${ }^{29} \mathrm{Si}^{-1}{ }^{1} \mathrm{H}$ correlation spectra), while monitoring the temporal crystallization of DDR. ${ }^{29} \mathrm{Si}-{ }^{1} \mathrm{H}$ NMR reveals that template molecules accommodated within the cages are sticking to these 8-ring windows through their amine group. High quality DD3R crystals are applied in the adsorptive separation of buta-1,3-diene and but-2-ene isomers, one of the most energy intensive separations in chemical industry. Mixture separation experiments revealed that the 8-ring apertures of the DD3R cages are only accessible to trans-but-2-ene and buta-1,3-diene, while excluding but-1-ene and cis-but-2-ene molecules, resulting in shape-selective separation in the presence of $\mathrm{C} 4$ mixtures.
\end{abstract}

\section{Introduction}

Zeolites are microporous aluminasilicates widely employed in chemical industry as molecular sieves and catalysts. ${ }^{1-6}$ The combination of high surface area, high adsorption capacity and pores of molecular dimensions in highly stable crystalline structures $^{2}$ makes this special class of aluminosilicates (zeolites) the solid of choice in many catalytic and separation processes.

Decadodecasil 3R (DDR) is formed by combining 10 (deca) hedron $\left[4^{3} 5^{6} 6^{1}\right]$ with 12 (dodeca) hedron $\left[5^{12}\right]$ cages yielding the only accessible cage, 19 hedron $\left[4^{3} 5^{12} 6^{1} 8^{3}\right]$. DDR belongs to the porosils ( $\mathrm{SiO}_{2}$ polymorphs), and further to zeosils (the expression "zeolite" has nowadays a broader meaning, to include all microporous silica-based solids presenting crystalline walls, including those materials where a fraction of $\mathrm{Si}$ atoms has been substituted by another element, $\mathrm{T}$, in the case of zeosils, $\mathrm{Si} / \mathrm{T}$ is larger than 500; these compounds are essentially Si-based, but, contrary to clathrasils, the porosity of these materials is accessible) as a consequence of its channel-like pore geometry and 8 -membered ring $(8 \mathrm{R})$ windows. ${ }^{7}$ These $8 \mathrm{R}$ pore openings enable

${ }^{a}$ Catalysis Engineering, Chemical Engineering Department, Delft University of Technology, Julianalaan 136, 2628 BL Delft, The Netherlands.E-mail: c.gucuyener@tudelft.nl; j.gascon@tudelft.nl

${ }^{b}$ Inorganic Materials Chemistry, Department of Chemical Engineering and Chemistry, Eindhoven University of Technology, 5600 MB Eindhoven, The Netherlands only small molecules to adsorb, which makes DDR a very attractive size selective adsorbent.

Several materials with the DDR topology have been reported: Sigma- $1,{ }^{8}$ DD3R ${ }^{9}$ and ZSM-58. ${ }^{10}$ Sigma-1 is the aluminiumcontaining version of DD3R, requiring adamantylamine as the structure directing agent (SDA), whereas ZSM-58 is synthesized using a different SDA, methyltropiniumiodide. ZSM-58 can be synthesized with $\mathrm{Si} / \mathrm{Al}$ ratios varying between 30 and $\infty .{ }^{11}$ The challenge in the synthesis of DD3R is its poor reproducibility. Formation of competitive phases like D1H (DOH) and Sigma-2 (SGT) under similar synthesis composition might also occur. These competitive crystalline products do not only decrease the purity of the desired phase, but they can also stick to the surface of the synthesis containers, acting as seed crystals and hindering future syntheses of DD3R, resulting in the so-called 'memory effect'. ${ }^{12}$

In the last decade we have demonstrated that materials with the DDR topology show a great potential in the fields of separation and catalysis. ${ }^{13-21}$ Regarding adsorptive based separations, the selective adsorption of propylene over propane and trans-but-2-ene and buta-1,3-diene over other butane and but-2ene isomers, together with the high uptakes of ethylene on the DD3R, was firstly reported by our group when studying single component adsorption using a Tapered Element Oscillating Microbalance (TEOM). ${ }^{22,23}$ Later, the separation of propane/ propylene mixtures was studied in detail by breakthrough 
experiments, which demonstrated the shape selective adsorption behaviour of DD3R for propylene. ${ }^{13}$

As a result of the close boiling points of unsaturated $\mathrm{C} 4$ isomers, their separation is among the most energy intensive processes in chemical industry. Usually a combination of separation methods is applied. The most difficult steps in the whole process are the extractive separation of buta-1,3-diene from but1-ene and the consecutive separation of but-1-ene from but-2enes, as high-purity but-1-ene is needed in the production of polymers. ${ }^{24}$ In addition, the possible separation of both cis and trans but-2-enes isomers is of importance for the production of high performance polymers.

The synthesis of DD3R was firstly reported by Gies ${ }^{9}$ in 1986 and later optimized and scaled up by den Exter et al. ${ }^{25}$ enabling a better characterization of the material. However, this synthesis required long synthesis times, typically from 25 to 42 days. Gascon et al. ${ }^{13}$ reduced the synthesis time to 2 days by the addition of DD3R seeds. Along the same line, Yajima and Nakayama ${ }^{26}$ decreased the amount of template and the amount of seed crystals in the synthesis of DD3R based membranes. Recently, Yang et al. ${ }^{27}$ reported the synthesis of DD3R in fluoride medium. Instead of the originally applied mobilizing agent ethylenediamine, KF was reported to drive the formation of pure phase DD3R. In the latter, the addition of seeds to the synthesis mixture reduced the synthesis time from 10 to 1 day.

The benefits of fluoride addition in the form of $\mathrm{HF}$ or $\mathrm{NH}_{4} \mathrm{~F}$ have been very often reported. ${ }^{28-41}$ Fluoride may act as a structure directing agent (SDA) and/or as a mineralizing agent (MA). When fluoride acts as a structure directing agent, ${ }^{28,40}$ it is proposed that fluoride tends to be placed at the smallest interstices available in the zeolite structure. The fluoride may yield negatively charged fluoro-silicate moieties that need to be compensated by the positively charged SDA molecules, like protonated alkylamines. Regarding the second, fluoride is thought to accelerate silanol condensation and thereby prevent the formation of $\mathrm{Si}-\mathrm{O}-\mathrm{Si}$ connection defects. ${ }^{30,33,34}$ Until the work of Yang et al., ${ }^{27}$ the synthesis of DD3R, normally carried out with a non-cationic template, in the presence of fluoride had not been reported before, while the actual role of the fluoride is not known yet.

In this work, special attention is paid to the reproducible and rapid synthesis of DD3R. Three synthesis methods were chosen for this assessment: Yajima and Nakayama, ${ }^{26}$ Gascon et al. ${ }^{13}$ and Yang et al. ${ }^{27}$ with a special emphasis on cleaning procedures to overcome the memory effects. To unravel the specific role of the addition of fluoride to the synthesis and to optimize synthesis conditions, MAS NMR in combination with reaction-composition studies has been carried out. Finally the potential of the produced high quality DD3R material in the adsorptive separation of buta-1,3-diene and but-2-ene isomer mixtures is explored.

\section{Experimental}

\subsection{Synthesis}

The general route for the synthesis of DD3R starts with the dissolution of the structure directing agent, SDA (adamantylamine), in the mobilizing ${ }^{42} /$ mineralizing agent. ${ }^{43}$ Following, the silica source and water are added and the synthesis is carried out at temperatures around $160-170{ }^{\circ} \mathrm{C} .{ }^{9,13,25,26}$ A summary of the different synthesis routes adapted from the literature ${ }^{13,26,27}$ is presented in Table 1 and Fig. 1. All chemicals were purchased from Sigma-Aldrich and used without further purification. SnowTex ${ }^{\circledR}$ was directly purchased from Nissan Chemical USA. Seed crystals used in every synthesis were prepared according to the method described by den Exter et al. ${ }^{25}$ For each recipe, the seed amount added corresponds to $0.1 \mathrm{wt} \%$ of the batch prepared. After each synthesis the solution is filtered to recover the powder and washed until neutral $\mathrm{pH}$. This step is followed by the cleaning with methanol to remove possible organic impurities.

\subsection{Memory effects}

'Memory effects' arise when the synthesis vessels are reused. If some product residue from previous syntheses stays on the autoclave liners' walls, it can act as seed and drive the synthesis towards the formation of undesired products. Different cleaning methods are practised to avoid memory effects. These cleaning procedures include aqueous solutions of $\mathrm{NaOH}, \mathrm{HF}$ and $\mathrm{KOH}$ with the molarities shown in Table 2 and each step is applied once overnight. Cleaning with $\mathrm{NaOH}$ is only carried out at high temperatures while with $\mathrm{KOH}$ both room temperature (RT) and high temperature (HT) conditions are practised. Cleaning with $\mathrm{HF}$ at high temperatures is not desired in view of the high toxicity of the acid, and thus only cleaning at low temperature is studied. In addition, due to adverse effects of HF to the synthesis, ${ }^{27}$ after cleaning at room temperature with $\mathrm{HF}$, the next day the polytetrafluoroethylene (PTFE) liner is also cleaned with water under the same conditions. Apart from the memory effect experiments, after every synthesis the PTFE liners used were cleaned with $1 \mathrm{M}$ $\mathrm{KOH}$ at a temperature higher than the synthesis temperature $\left(\geq 10^{\circ} \mathrm{C}\right)$ under rotation.

\subsection{Characterization}

The crystalline structure of the powders was analyzed by X-ray diffraction (XRD) using a Bruker-AXS D5005 with $\mathrm{CuK} \alpha$ radiation.

Thermogravimetric analysis (TGA) was performed by means of a Mettler Toledo TGA/SDTA851e, on $10 \mathrm{mg}$ samples in an air flow of $100 \mathrm{ml} \mathrm{min}^{-1}$ at a heating rate of $10 \mathrm{~K} \mathrm{~min}^{-1}$ up to $1173 \mathrm{~K}$.

Scanning Electron Microscopy (SEM) was measured in a JEOL JSM 6500F setup coupled to an Energy Dispersive Spectrometer (EDS) for micro-analysis.

Nitrogen adsorption at $77 \mathrm{~K}$ in a Quantachrome Autosorb-6B unit gas adsorption analyzer was used to determine the textural properties at the BET surface area between 0.05 and 0.15 relative pressures and pore volume at 0.95 relative pressure.

${ }^{13} \mathrm{C}$ (MAS) NMR spectroscopy for the determination of the protonation state of adamantylamine was performed on a Bruker Avance- 400 with a $5 \mathrm{~mm}$ zirconia rotor and a spinning frequency of $11 \mathrm{kHz}$. Rest of the MAS NMR spectra were recorded on a Bruker DMX 500 spectrometer operating at ${ }^{1} \mathrm{H}$, ${ }^{19} \mathrm{~F}$ and ${ }^{29} \mathrm{Si}$ NMR frequencies of 500, 471 and $99 \mathrm{MHz}$, respectively. For ${ }^{19} \mathrm{~F}$ MAS NMR the proton channel of a $2.5 \mathrm{~mm}$ MAS probe head was tuned to the ${ }^{19} \mathrm{~F}$ NMR frequency. Single-pulse 
Table 1 Comparison of the three methods used with respect to synthesis conditions, compositions and reactants

\begin{tabular}{llll}
\hline & Yajima et al. Method 1 & Gascon et al. Method 2 & Yang et al. Method 3 \\
\hline $\begin{array}{l}\text { Silica source } \\
\begin{array}{l}\text { Mineralizing agent } \\
\text { (MA) source }\end{array}\end{array}$ & $\begin{array}{l}\text { Snowtex-30 } \\
\text { Ethylenediamine (EDA) }\end{array}$ & $\begin{array}{l}\text { Aerosil 200 } \\
\text { Ethylenediamine (EDA) }\end{array}$ & $\begin{array}{l}\text { Ludox-30 } \\
\text { Potassium fluoride } \\
\text { dihydrate (KF.2H }\end{array}$ \\
$\begin{array}{l}\text { Synthesis molar composition } \\
\left(\mathrm{ADA}: \mathrm{SiO}_{2}: \mathrm{MA}: \mathrm{H}_{2} \mathrm{O}\right)\end{array}$ & $2.68: 100: 1600: 2400$ & $47: 100: 404: 11240$ & $47: 100: 100: 8000$ \\
$\mathrm{MA} / \mathrm{ADA}$ & 16 & 8.60 & 2.13 \\
$\mathrm{ADA} / \mathrm{SiO}_{2}$ & 0.03 & 0.47 & 0.47 \\
$\mathrm{H}_{2} \mathrm{O} / \mathrm{SiO}_{2}$ & 24 & 112.4 & 80 \\
$\begin{array}{l}\text { Synthesis temperature/K } \\
\text { Synthesis time/h }\end{array}$ & 438 & 433 & 443 \\
\end{tabular}

excitation with a $90^{\circ}$ pulse of $2.5 \mu$ s was applied and the samples were rotated at a rate of $15 \mathrm{kHz}$. The relaxation delay between subsequent NMR scans was $600 \mathrm{~s}$, which is 1.3 times the spinlattice relaxation time $T_{1} \approx 450 \mathrm{~s}$ of crystalline $\mathrm{KF}$. Because of the long $T_{1}$ time of $\mathrm{KF}$, polytetrafluoroethylene (PTFE) was used as an external reference for pulse and chemical-shift calibration $\left(\delta=-124 \mathrm{ppm}\right.$ with respect to $\mathrm{CFCl}_{3}$ (ref. 44)). Proton decoupled ${ }^{29} \mathrm{Si}$ MAS NMR spectra were recorded by use of a $4 \mathrm{~mm}$ MAS probe head and a sample-rotation rate of $5 \mathrm{kHz}$. To suppress baseline artefacts arising from probe ringing, directexcitation ${ }^{29} \mathrm{Si}$ NMR spectra were recorded using a Hahn-echo pulse sequence $90^{\circ}-\tau-180^{\circ}-\tau$, two pulse sequence with a $90^{\circ}$ pulse of $5 \mu \mathrm{s}$, and a delay $\tau=2.5 \mu \mathrm{s}$. One-dimensional ${ }^{1} \mathrm{H}-{ }^{29} \mathrm{Si}$ crosspolarization spectra were recorded with a ramp-shaped proton pulse combined with a rectangular ${ }^{29} \mathrm{Si}$ pulse of $10 \mathrm{~ms}$ and an interscan delay of $5 \mathrm{~s}$. Two-dimensional ${ }^{1} \mathrm{H}-{ }^{29} \mathrm{Si}$ correlation spectra were recorded with a rectangular pulse of $3 \mathrm{~ms}$ with carefully matched amplitudes on both channels. Tetramethylsilane (TMS) was employed as an external reference for the chemical shift for ${ }^{1} \mathrm{H}$ and ${ }^{29} \mathrm{Si} \mathrm{NMR}$.

\subsection{Adsorptive separation}

Breakthrough experiments were carried out using a column with a length of $5 \mathrm{~cm}$ and an outer diameter of $1 / 4 \mathrm{in}$, packed with
Table 2 Details of the cleaning techniques used (+ or - represents whether it is applied or not)

\begin{tabular}{llll}
\hline & $\mathrm{NaOH}$ & $\mathrm{HF}$ & $\mathrm{KOH}$ \\
\hline Molarity of the solution & 1 & 0.1 & 1 \\
Cleaning at room temperature & - & + & + \\
Cleaning at high temperature $\left(190^{\circ} \mathrm{C}\right)$ & + & - & + \\
Cleaning with water at room temperature & - & + & - \\
\hline
\end{tabular}

$320 \mathrm{mg}$ of pelletized $\left(5\right.$ ton $\mathrm{cm}^{-2}$ ) sample (particle size between 500 and $710 \mu \mathrm{m}$ ). More details about the experimental setup can be found elsewhere. ${ }^{45}$ In situ activation of the adsorbent was performed in a pure $\mathrm{He}$ flow of $0.616 \mathrm{mmol} \mathrm{min}^{-1}$ at $473 \mathrm{~K}$ for $10 \mathrm{~h}$.

Gases were provided by HoekLoos (minimum purity $99.95 \%$ ). Gas mixtures were prepared via mass flow controllers. The residence time of the empty volume in the system (tubing, interparticle space) is calculated by a separate experiment using both but-1-ene and helium as the reference components and used to correct the breakthrough time and mass balance calculations.

The outlet of the column was analyzed by means of a gas chromatograph (GC). Hydrogen breakthrough experiments were carried out to determine the system volume. In this case the outlet flows were analysed using a mass spectrometer (MS). Since

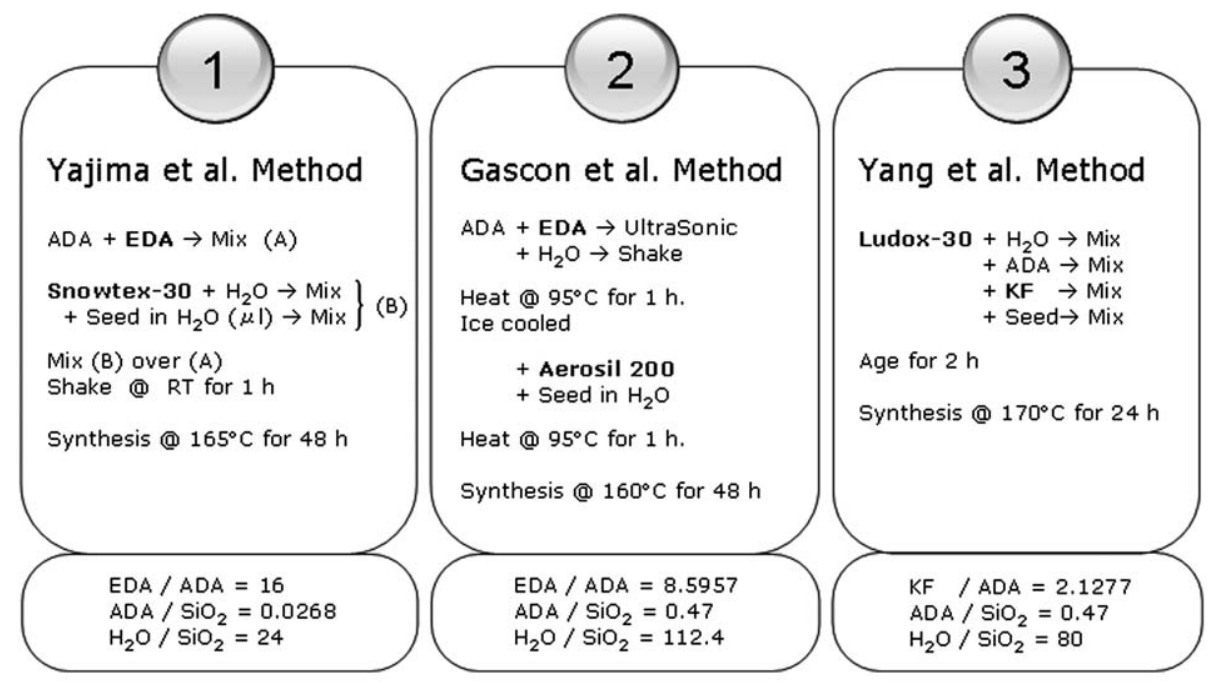

EDA and ADA stand for ethylenediamine and adamantylamine, respectively.

Fig. 1 Synthesis procedure used for the three different methods ${ }^{13,26,27}$ in this study. 
during the experiments pure hydrocarbon mixtures are fed to the column, throughout the adsorption process the exit flow rate of the column may change from zero (complete adsorption) to the inlet flow rate. Hence, a constant flow of helium $(4.10 \mathrm{mmol}$ $\mathrm{min}^{-1}$ ) in excess of the inlet flow was added immediately at the outlet of the column. In view of this excess the measured GC intensities are proportional to the molar flows of the components leaving the column.

Breakthrough experiments were performed at different temperatures with $0.178 \mathrm{mmol} \mathrm{min}{ }^{-1}$ total flow of hydrocarbons. In the figures, breakthrough curves are presented in the form of component exit molar flow rates normalized by their inlet molar flow rate. The adsorbed amounts, $q_{\mathrm{i}}$ and $q_{\mathrm{j}}$, were calculated from a mass balance over the flow versus time data.

After every breakthrough experiment, a temperature programmed desorption (TPD) was monitored in order to assess the regenerability of the adsorbent. In such an experiment the concentration of the adsorbates at the exit of the column in a purge flow of pure helium $\left(4.46 \mathrm{mmol} \mathrm{min}^{-1}\right)$ is followed.

\section{Results}

\subsection{Assessment of different synthesis methods}

The three synthesis methods investigated in detail in this study will be referred to as Methods $1,{ }^{26} 2,{ }^{13}$ and $3 .{ }^{27}$ Reproducibility is defined as the possibility of obtaining DD3R several consecutive

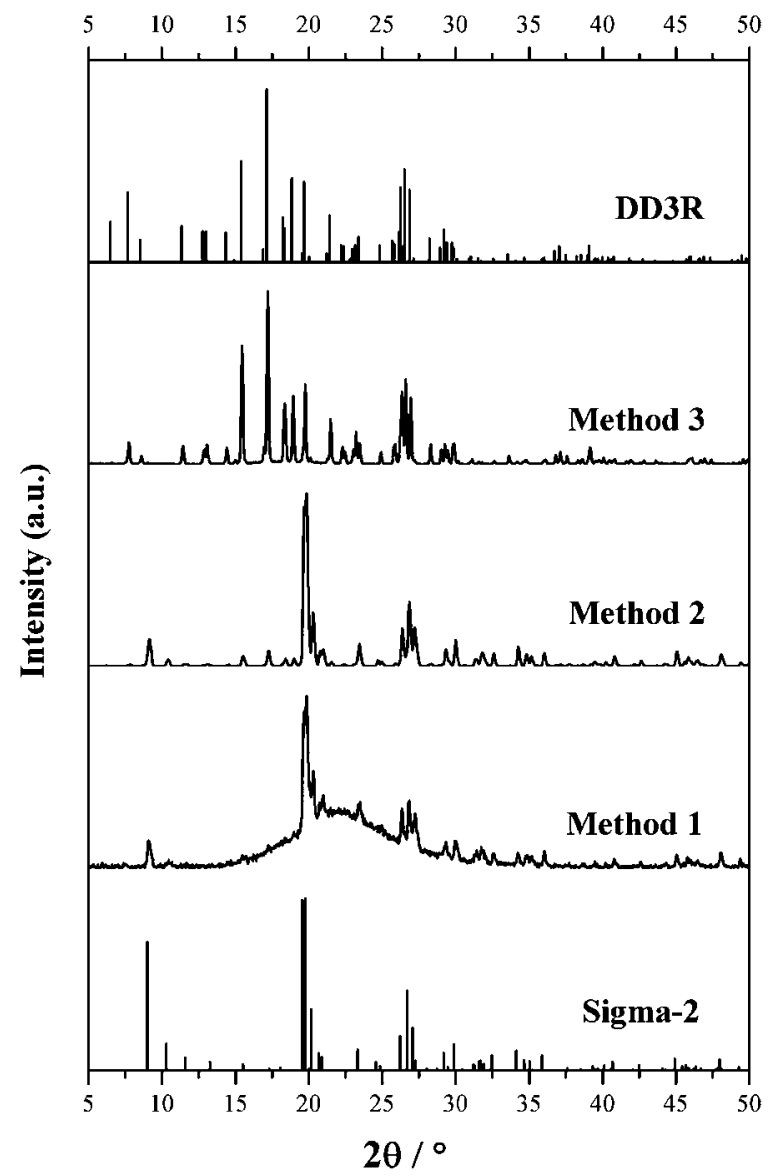

Fig. 2 XRD patterns of the powders synthesized by using three different methods, compared with the SGT and DDR powder patterns. syntheses in the same PTFE vessel. At least three different batch syntheses are carried out for each method to check the reproducibility. In Fig. 2, representative XRD patterns of the powders obtained from these three different methods are given and compared to that of the DDR and SGT topologies. ${ }^{46}$ From the batches synthesized using Method 1, two out of three syntheses resulted in Sigma-2, while the other resulted in a mixture of amorphous material and Sigma-2. Method 2 yielded varying batches with either DD3R or Sigma-2. In contrast, every synthesis using Method 3 always resulted in pure DD3R.

\subsection{Memory effects}

Memory effects arise when the synthesis vessels are reused. If some product residue from previous synthesis stays on or within the autoclave liners' walls, it can act as seed and drive the synthesis towards the formation of undesired products. Although cleaning methods are not extensively reported in the literature, in the case of DD3R synthesis it became an essential aspect. In this section Method 3 is used to outline the main problems found during this research.

One of the autoclave liners used for the synthesis of DD3R with Method 1 yielded Sigma-2 crystals during the following syntheses using Method 3. Thus, the autoclave liner is considered as contaminated. This PTFE liner was then cleaned following the various procedures and used in the synthesis of DD3R with Method 3 after every cleaning method. Fig. 3 shows the XRD

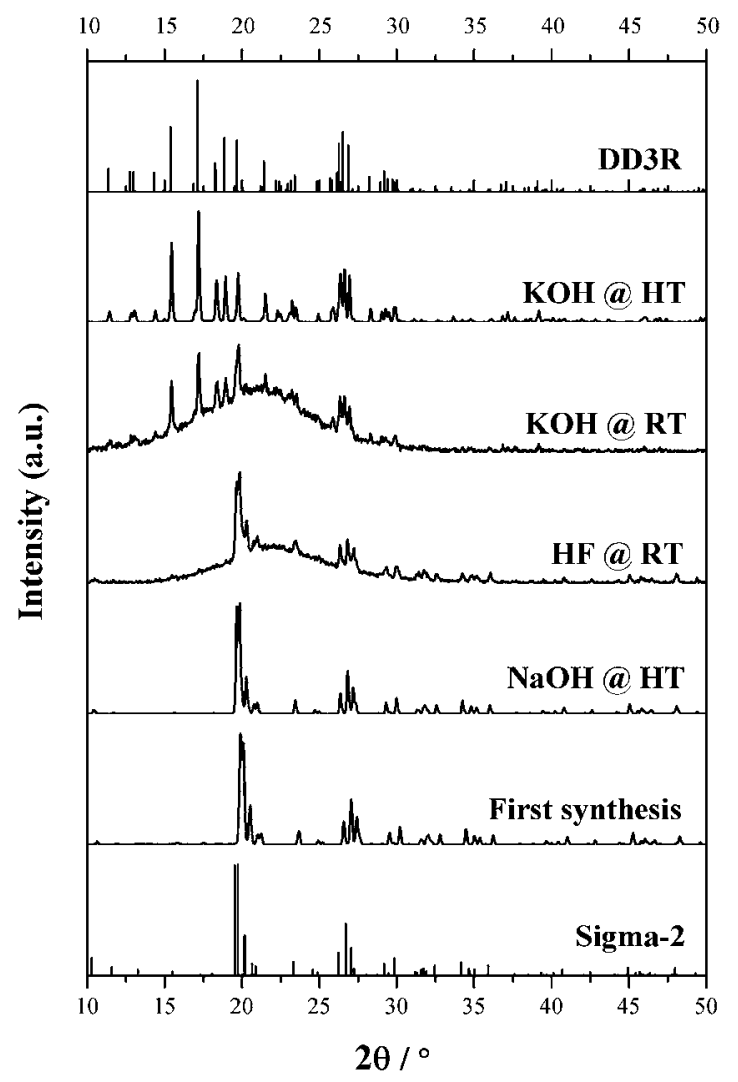

Fig. 3 XRD patterns of the powders obtained according to Method 3 for $10 \mathrm{~h}$, after different cleaning methods of the PTFE liners used after a first synthesis. SGT (sigma-2) and DDR (DD3R) patterns are included for comparison. 
patterns of the products that are formed in the PTFE liner after 24 hours of synthesis, in comparison to the first synthesis that yielded Sigma-2 crystals using Method 1. Synthesis carried out after cleaning with $\mathrm{NaOH}$ at high temperature showed the same SGT pattern as the one without cleaning, indicating no apparent cleaning effect. In the case of $\mathrm{HF}$, on the other hand, the following synthesis caused most of the product to be amorphous and only when the autoclave liner is treated with $\mathrm{KOH}$ under autogenous pressure at elevated temperature pure phase DD3R is formed.

To show the destructive effect of $\mathrm{KOH}$, a synthesis was carried out with a molar composition of $\mathrm{ADA}: \mathrm{SiO}_{2}: \mathrm{KF}: \mathrm{KOH}$ : $\mathrm{H}_{2} \mathrm{O}=47: 100: 100: 100: 8000$ using Sigma-2 as seed $(3 \mathrm{wt} \%$ of the solution prepared) following Method 3. SEM pictures of the crystals obtained after the synthesis are given in Fig. 4. The crystals contained holes over the surface and most of them had lost their integrity.

\subsection{Advances in the fluoride route}

Fluoride addition is mostly studied for zeolite syntheses where cationic templates are involved. In order to check whether addition of fluoride to the synthesis causes protonation of the organic template, ${ }^{13} \mathrm{C}$ (MAS) NMR spectroscopy has been applied to characterize the protonation state of the adamantylamine template on the as synthesized DD3R sample. The ${ }^{13} \mathrm{C}$ NMR spectrum (Fig. 5) shows only the free amine: the signal of the carbon atom ${ }^{13} \mathrm{C}$ carrying the amine group gives a shift located at $\sim 43$ ppm (peak $A$ in Fig. 5), whereas the protonated ammonium state would give rise to a signal at approximately 55 ppm.

${ }^{19} \mathrm{~F}$ NMR spectra were collected from both as synthesized and calcined DD3R samples as given in Fig. 6. The observed chemical shift, $-134 \mathrm{ppm}$, is close to the values -132 and $-133 \mathrm{ppm}$, previously reported shifts of KF. ${ }^{47,48}$ Neither by recording $192{ }^{19} \mathrm{~F}$ NMR scans of as-prepared DD3R with an interscan delay of $600 \mathrm{~s}$ during 32 hours, nor by accumulating 1024 scans with an interscan delay of $30 \mathrm{~s}$, any ${ }^{19} \mathrm{~F}$ NMR signal was detected. No signal from, e.g., residual $\mathrm{KF}$ at $-134 \mathrm{ppm}$, nor from $\mathrm{F}^{-}$ anions within the cages around $-180 \mathrm{ppm}^{49}$ have been observed.

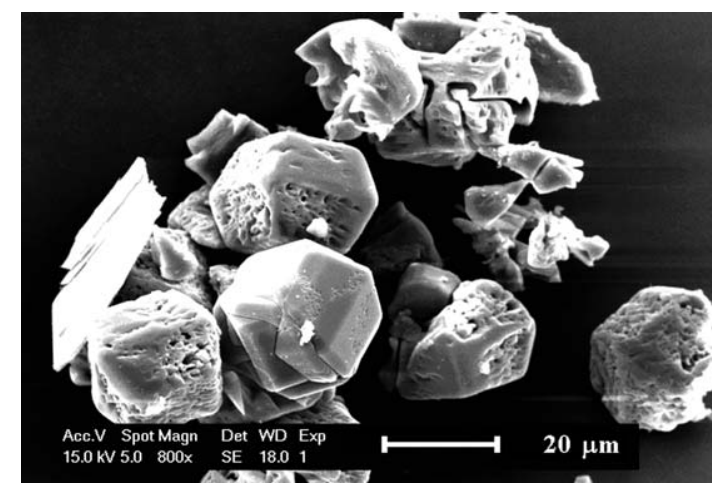

Fig. 4 SEM micrograph of the powder left from the synthesis with a composition of $\mathrm{ADA}: \mathrm{SiO}_{2}: \mathrm{KF}: \mathrm{KOH}: \mathrm{H}_{2} \mathrm{O}=$ $47: 100: 100: 100: 8000$ and Sigma-2 was used as seed $\left(3 \mathrm{wt}^{\%} \%\right.$ of the solution prepared) following Method 3.

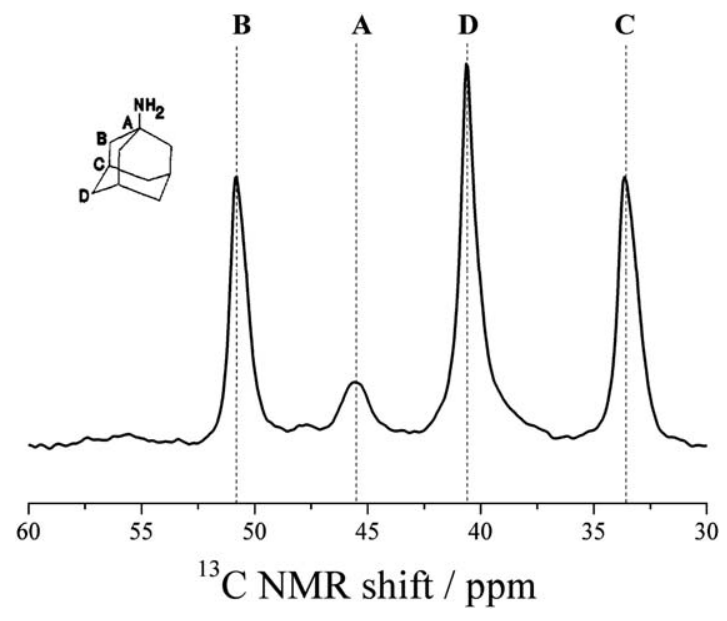

Fig. $5{ }^{13} \mathrm{C}$ MAS NMR analysis of DD3R powder synthesized using Method 3 (24 h).

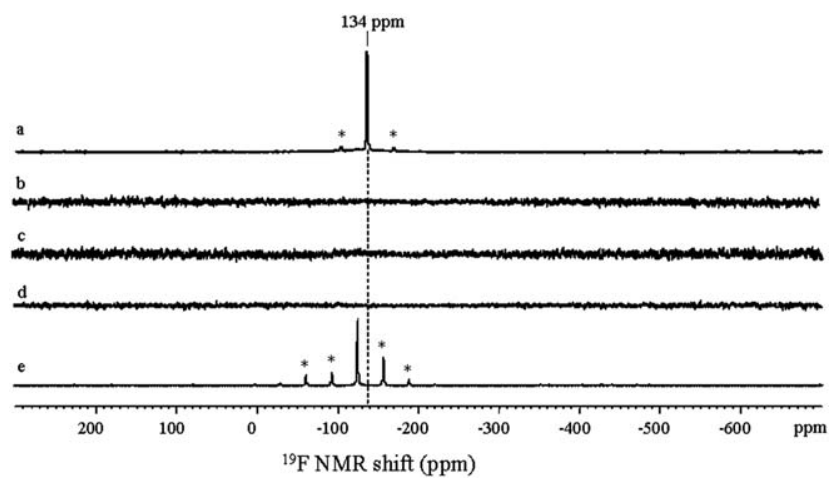

Fig. $6{ }^{19} \mathrm{~F}$ MAS NMR spectra of (a) KF (10.6 mg; 4 scans; $600 \mathrm{~s}$ interscan delay), (b) as-prepared DD3R (7.7 mg, 192 scans, $600 \mathrm{~s})$, (c) asprepared DD3R (7.7 mg, 1024 scans, $30 \mathrm{~s})$, (d) calcined DD3R (6.3 mg, 128 scans, $30 \mathrm{~s}$ ), and (e) polytetrafluoroethylene (16.5 mg; 4 scans, $5 \mathrm{~s}$ ). All samples were measured at $298 \mathrm{~K}$. The sample rotation rate was $15 \mathrm{kHz}$. Spinning sidebands are indicated with an asterisk.

In order to proof differences in the local chemical environment of silicon, ${ }^{50}{ }^{29} \mathrm{Si}$ (MAS) NMR spectra of the DD3R as synthesized and calcined crystals were recorded (Fig. 7). In the spectrum of calcined DD3R (Fig. 7d) seven signal components of NMR-distinct silicon sites can be recognized (Fig. 7c). The different chemical shifts of these $\mathrm{Q}^{4}$ signals arise from $\mathrm{O}-\mathrm{Si}-\mathrm{O}$ bond-angle variations. There are no signals in the typical $\mathrm{Q}^{3}$ (corresponding with $\left.\mathrm{Si}-(\mathrm{OSi})_{3} \mathrm{OH}\right)$ range $(90-100 \mathrm{ppm})$, as expected for a defect-free silicate structure. At room temperature the ${ }^{29} \mathrm{Si}$ NMR signals of the as-prepared DD3R still containing the adamantylamine (Fig. 7b and c) are broader than those of the calcined zeolite (Fig. 7d). The signals between -110 and -115 ppm do not only broaden, but also shift further downfield. This reflects chemical-exchange broadening resulting from fluctuating template-zeolite interactions, because at $233 \mathrm{~K}$ it splits into a signal at -111 and $-113 \mathrm{ppm}$ (Fig. 7a). The latter position practically coincides with that of the four overlapping down-field signals of the calcined zeolite without template. Similar to the major signal on the downfield side, the minor signal on the upfield side shows signs of exchange broadening at room 


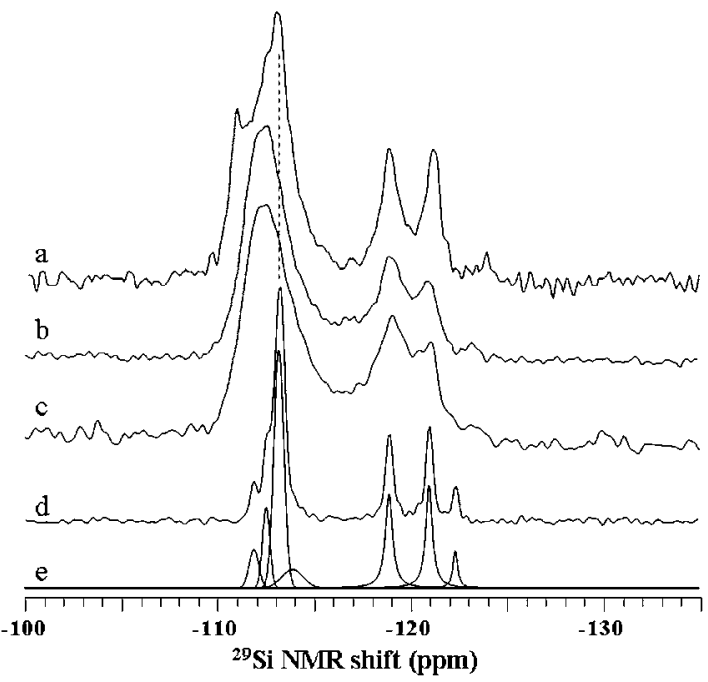

Fig. $7{ }^{29} \mathrm{Si}$ MAS NMR spectra of (a-c) as-prepared and (d) calcined DD3R. (a and b) Cross-polarization spectrum at (a) $233 \mathrm{~K}$ and (b) $296 \mathrm{~K}$; (c and d) direct-excitation spectra at $296 \mathrm{~K}$; (e) decomposition of the calcined DD3R spectrum (d) into seven lineshape components.

temperature. At $233 \mathrm{~K}$ a narrow signal appears at $-123.5 \mathrm{ppm}$, which is about $1 \mathrm{ppm}$ shifted relative to the corresponding signal of calcined DD3R at $-122.3 \mathrm{ppm}$.

To investigate the template-zeolite interaction in more detail, two-dimensional (2D) ${ }^{29} \mathrm{Si}^{-1} \mathrm{H}$ correlation spectra ${ }^{51}$ of the assynthesized DD3R crystal were recorded (Fig. 8). This experiment is based on the dipole coupling between the ${ }^{1} \mathrm{H}$ spins in adamantylamine and the ${ }^{29} \mathrm{Si}$ spins in the DD3R lattice, which is a measure of their distance $(<5 \AA)$. This coupling is weak, and cross-polarization is only feasible at moderate sample-rotation rates $\leq 5 \mathrm{kHz}$. At such low MAS rate the ${ }^{1} \mathrm{H}$ NMR resolution is limited compared to ${ }^{1} \mathrm{H}$ NMR spectra at MAS rates $\geq 10 \mathrm{kHz}$ (Fig. 8b). At $10 \mathrm{kHz}$ MAS the ${ }^{1} \mathrm{H}$ NMR spectrum shows three signals at 2.1, 1.6 and $0.8 \mathrm{ppm}$, which can be respectively assigned to methine $(\mathrm{CH})$, methylene $\left(\mathrm{CH}_{2}\right)$ and amino $\left(\mathrm{NH}_{2}\right)$ moieties in adamantylamine. Methine protons are relatively underrepresented in the 2D correlation spectrum (Fig. $8 \mathrm{~b}$ and c) and are

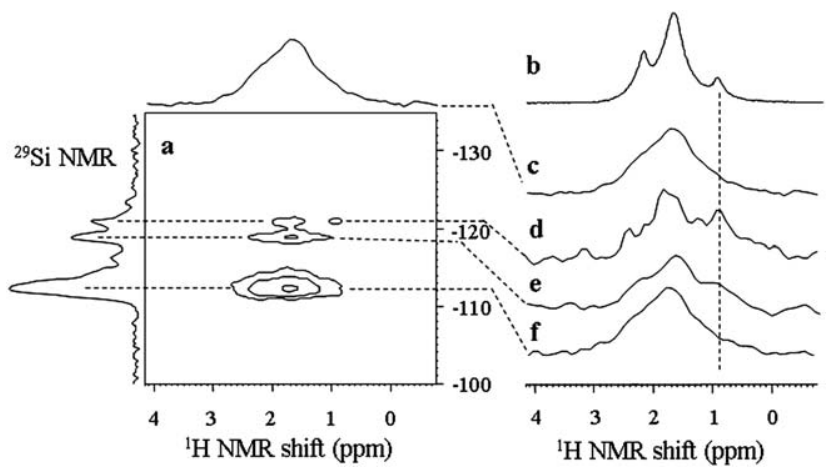

Fig. 8 (a) Two-dimensional ${ }^{1} \mathrm{H}^{29} \mathrm{Si}$ correlation spectrum with projections on the direct ${ }^{29} \mathrm{Si}$ NMR axis (vertical) and indirect ${ }^{1} \mathrm{H}$ NMR axis (horizontal). (b) ${ }^{1} \mathrm{H}$ MAS NMR spectrum at double sample rotation rate, $10 \mathrm{kHz}$; (c) projection on the ${ }^{1} \mathrm{H}$ NMR axis; ${ }^{1} \mathrm{H}$ NMR cross-sections at constant ${ }^{29} \mathrm{Si}$ NMR shifts (d) $-120.9 \mathrm{ppm}$, (e) -118.8 and (f) $-112.3 \mathrm{ppm}$. The 2D spectrum was recorded at a MAS rate of $5 \mathrm{kHz}$. probably relatively distant from the nearest silicon atom in the DD3R lattice. The major ${ }^{29} \mathrm{Si}$ NMR signal with its effective maximum at $-112.3 \mathrm{ppm}$ correlates mainly with methylene protons (Fig. 8f). Interestingly, the ${ }^{29} \mathrm{Si}$ NMR signals at -120.9 and $-118.8 \mathrm{ppm}$ show a correlation with the amino signal at 0.8 ppm (Fig. 8d and e). A particularly clear correlation is observed for the $-120.9 \mathrm{ppm}$ signal, which is assigned to silicon sites in the 8-membered rings connecting the cages, the windows. ${ }^{50}$ In addition to the correlation with amino moieties, there is also a correlation between the ${ }^{29} \mathrm{Si}$ NMR signal at $-120.9 \mathrm{ppm}$ and the methylene signal at $1.6 \mathrm{ppm}$. Thus, the overall picture from NMR is that the adamantylamine template molecules are accommodated inside the DD3R cages with the amino moieties sticking into the 8-membered rings of the windows. The template mobility reflected by the exchange broadening of the major signal at $-112.3 \mathrm{ppm}$ could be rotated about the axis perpendicular to the ring.

Zeolite synthesis usually proceeds through an S-shaped crystallization curve $e^{3,52,53}$ and such curve yields valuable information on the kinetics of formation. Therefore, the crystallization process was monitored by quenching various syntheses at different times. XRD patterns of samples collected after 8, 16 and 24 hours of synthesis are shown in Fig. 9.

The product collected from the autoclaves after $8 \mathrm{~h}$ synthesis is mostly amorphous, with already some crystalline DD3R. The picture changes after $16 \mathrm{~h}$; a mixture of both Sigma-2 and DD3R

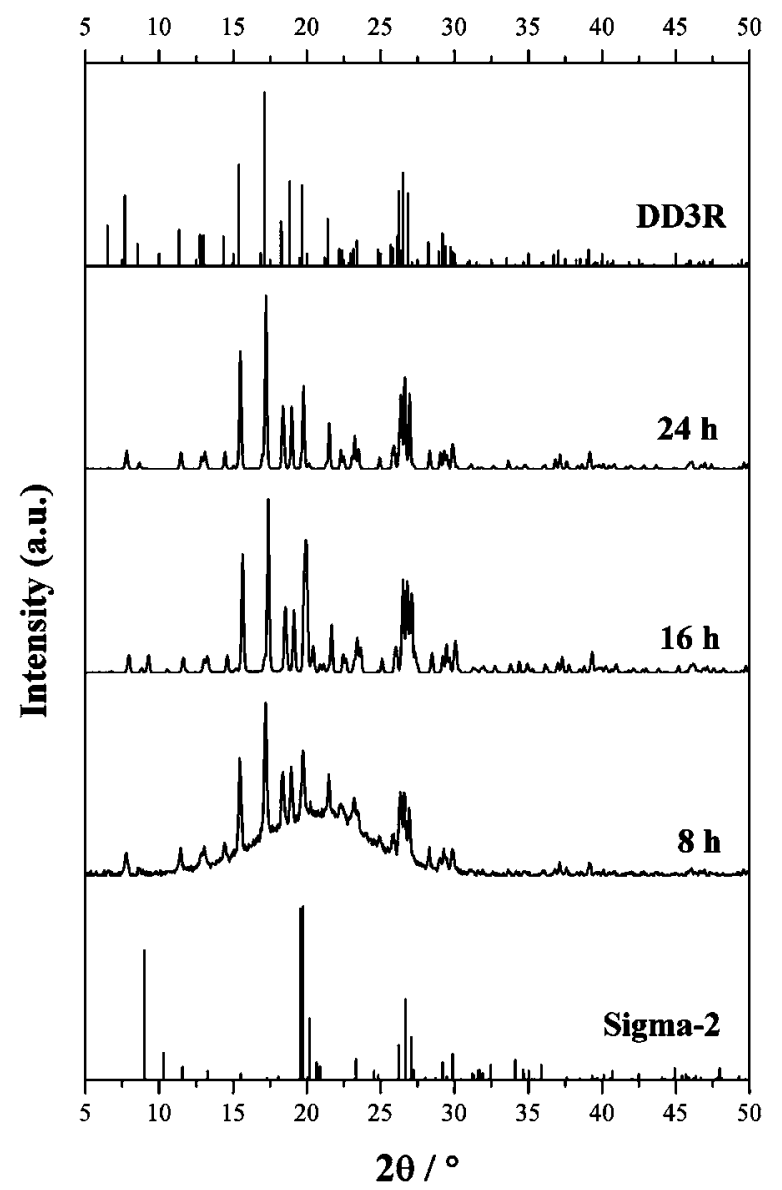

Fig. 9 XRD patterns of samples prepared by Method 3 for different times, compared with the SGT and DDR powder patterns. 
crystals is found. Interestingly, Sigma-2 crystals are not found after $24 \mathrm{~h}$. By increasing the synthesis time up to $48 \mathrm{~h}$, no substantial change in the pattern can be observed.

Crystal size and morphology were also monitored by SEM until the $24^{\text {th }}$ hour (Fig. 10). Apparently, under the studied synthesis conditions, the formation of both DD3R and Sigma-2 phases is promoted (Fig. 10a and b). However, the crystals of Sigma-2 $(10 \mu \mathrm{m})$ are much larger than those of DD3R $(2 \mu \mathrm{m})$, indicating a shorter induction period. By the $16^{\text {th }}$ hour, DD3R crystals cover a size range of 5-20 $\mu \mathrm{m}$, while intergrowths with some of the Sigma-2 crystals covering sizes of $20-30 \mu \mathrm{m}$ are observed (Fig. 10c and d). Details of these intergrown crystals (Fig. 11) reveal the formation of DD3R on top of them. After $24 \mathrm{~h}$ synthesis (Fig. 10e and f), the product looks more homogeneous. DD3R particles of $15-20 \mu \mathrm{m}$ and a few 20-25 $\mu \mathrm{m}$ agglomerates composed of $\sim 2 \mu \mathrm{m}$ DD3R crystals are observed. In agreement with the XRD results, hardly any Sigma-2 particle is observed after 24 hours of synthesis.

Different syntheses varying $\mathrm{KF} / \mathrm{ADA}$ and $\mathrm{KF} / \mathrm{SiO}_{2}$ ratios were carried out in order to find the compositional boundaries of formation for DD3R. Results are presented in a ternary diagram (Fig. 12). Apparently, if insufficient KF is present in the synthesis medium, pure DD3R is not formed.

To complete the full characterization of the samples, in Table 3 $\mathrm{N}_{2}$ adsorption and TGA results together with yield calculations from 3 different samples synthesized for 24 hours are given. Yield is calculated as grams of zeolite obtained per gram of silica source in the synthesis, while taking into account the amount of seed and template within the structure (eqn (1)). An average BET surface of $330 \mathrm{~m}^{2} \mathrm{~g}^{-1}$ and a yield of $80 \%$ with respect to the silica source are obtained.

$$
Y_{\mathrm{DDR}}=\frac{\left(m_{\text {product }}-m_{\text {seed }}\right)\left(\% \text { Relative Weight }_{\mathrm{TGA}}\right) / 100}{m_{\mathrm{SiO}_{2} \text { fed }}}
$$

\subsection{Separation performance}

The separation of mixtures containing but-1-ene, buta-1,3-diene, cis-but-2-ene and trans-but-2-ene on the synthesized DD3R has been investigated by means of multicomponent breakthrough experiments.
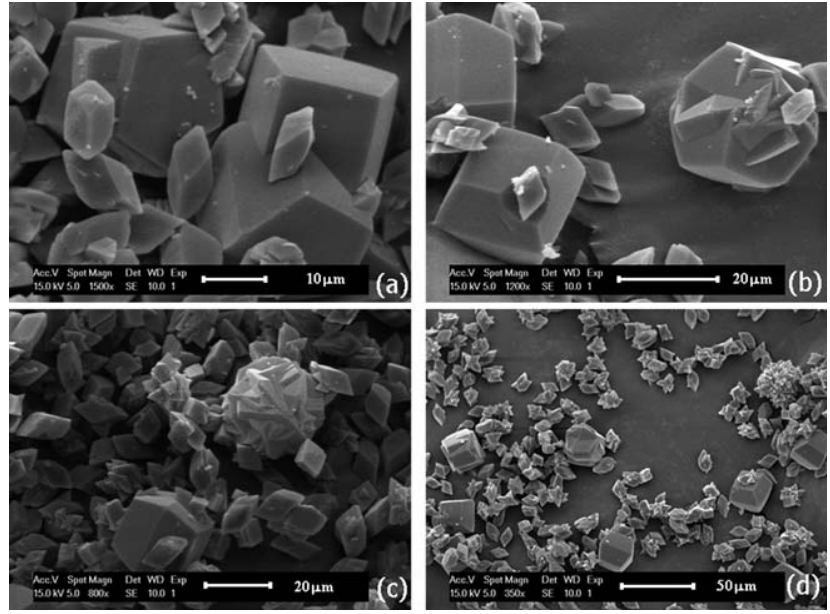

Fig. 11 SEM micrographs of the sample prepared by Method 3 for $16 \mathrm{~h}$, showing the intergrowth and transformation of Sigma-2 crystals to DD3R.

Fig. 13 shows the breakthrough profiles of but-1-ene, cis-but2-ene and trans-but-2-ene in a ternary mixture $(1: 1: 1)$ at $120 \mathrm{kPa}$ and two different temperatures: $303 \mathrm{~K}$ (a) and $373 \mathrm{~K}$ (b). Both but-1-ene and cis-but-2-ene do not adsorb significantly in DD3R and leave the bed first. Their molar fractions increase temporarily to 0.5 , which corresponds with their molar fraction in the feed after removal of trans-but-2-ene. In every case, but-1ene seems to break through slightly earlier than cis-but-2-ene. The absence of any roll-up implies that adsorption of these two components and displacement by trans-but-2-ene do not take place. After about $100 \mathrm{~s}$ at $303 \mathrm{~K}$, trans-but-2-ene breaks through. As concluded for the separation of propane and propylene,${ }^{13}$ internal diffusion in the DD3R crystallites is clearly rate determining, resulting only in a gradual increase of the transbut-2-ene concentration at the exit gas after breakthrough. The determined mixture adsorption selectivities for trans-but-2-ene/ cis-but-2-ene and trans-but-2-ene/but-1-ene are 3.5 and $3.7( \pm 0.5)$ at $303 \mathrm{~K}$, whereas they decrease to 3.0 and $2.8( \pm 0.5)$ at $373 \mathrm{~K}$, respectively.
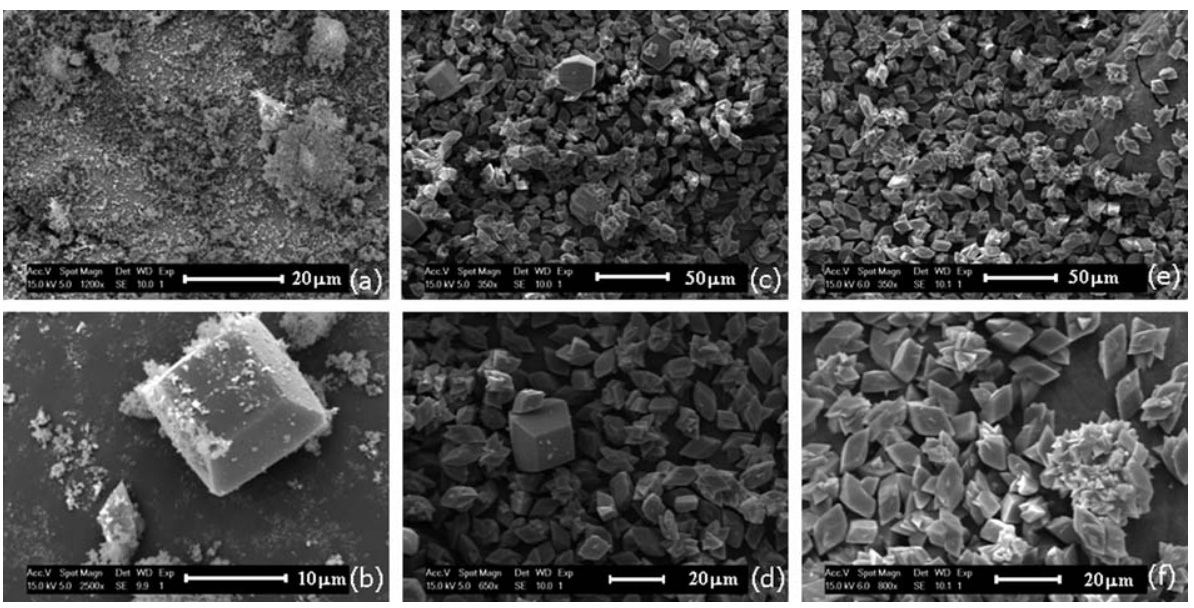

Fig. 10 SEM micrographs of the samples prepared by Method 3 for different times. 


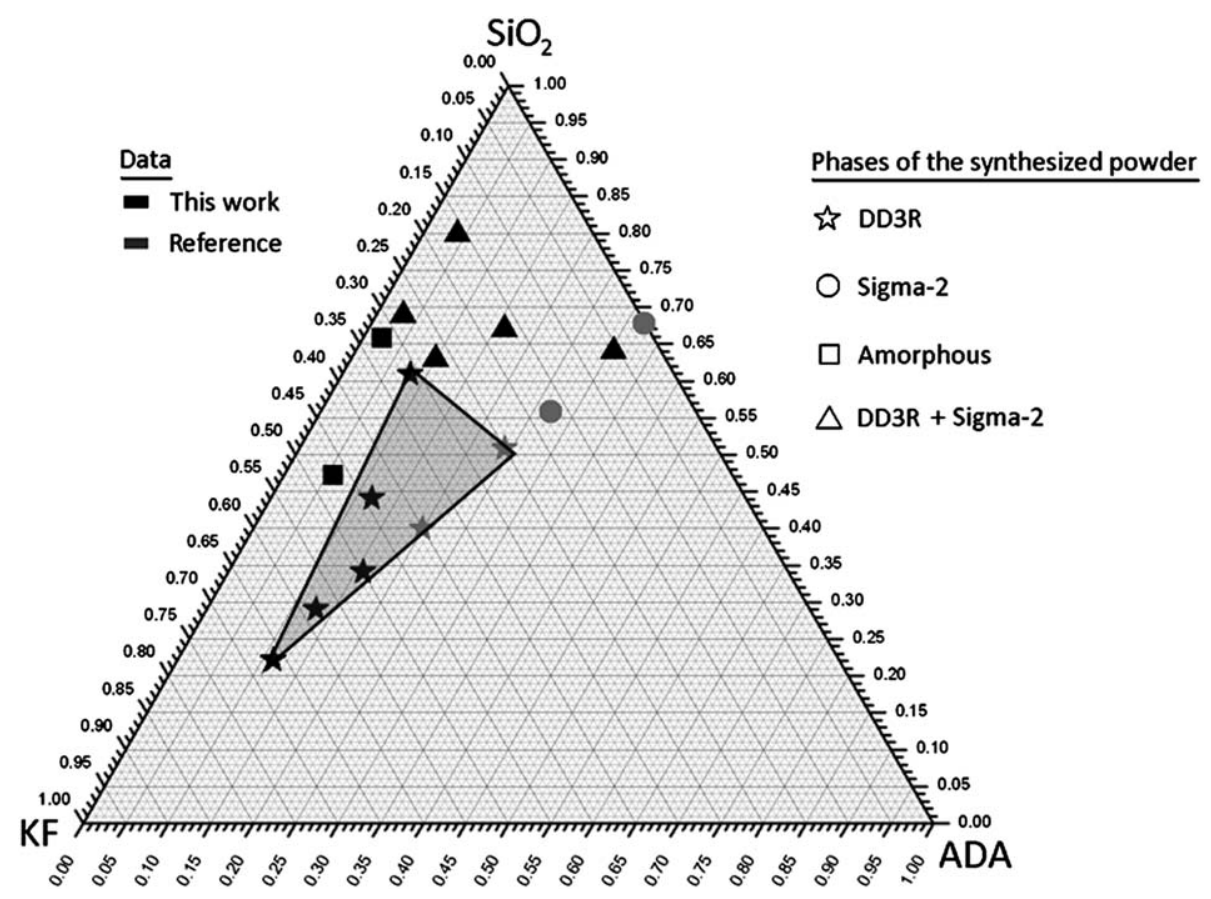

Fig. 12 Ternary phase diagram based on the liquid solution composition in the autoclave liners for the syntheses of DD3R crystals at $443 \mathrm{~K}$ for $24 \mathrm{~h}$ with the addition of seeds. Reference data are taken from Yang et al. where the syntheses are carried out at $443 \mathrm{~K}$ for 10 days without the addition of seeds. The $\mathrm{H}_{2} \mathrm{O}$ content of the liquid compositions is greater than $95 \mathrm{~mol} \%$. Corners: ADA represents the adamantylamine, $\mathrm{KF}$ represents the potassium fluoride, and $\mathrm{SiO}_{2}$ represents the silica.

Table 3 Specific surface area and pore volume from nitrogen adsorption at $77 \mathrm{~K}$, detemplation weight loss from TG analysis in air, and DD3R yields for three syntheses according to Method 3

\begin{tabular}{|c|c|c|c|c|}
\hline & $\begin{array}{l}\text { BET surface } \\
\text { area } / \mathrm{m}^{2} \mathrm{~g}^{-1}\end{array}$ & $\begin{array}{l}\text { Micropore } \\
\text { volume } / \mathrm{cm}^{3} \mathrm{~g}^{-1}\end{array}$ & $\begin{array}{l}\text { Relative } \\
\text { weight } \\
\text { loss }(\%)\end{array}$ & $\begin{array}{l}\text { DD3R yield } \\
\text { ( } \mathrm{g} \text { product } \\
\left.\text { per } \mathrm{g} \mathrm{SiO}_{2}\right)\end{array}$ \\
\hline Sample 1 & 348 & 0.15 & 11 & 79 \\
\hline Sample 2 & 329 & 0.16 & 11 & 84 \\
\hline Sample 3 & 315 & 0.14 & 11 & 80 \\
\hline Average & $331 \pm 17$ & $0.15 \pm 0.1$ & 11 & $81 \pm 3$ \\
\hline
\end{tabular}

Similar experiments were performed with an equimolar buta1,3-diene/but-1-ene mixture (Fig. 14a and b). At every temperature, a clear selectivity towards buta-1,3-diene is observed. The breakthrough of bute-1,3-diene starts almost 150 seconds after the breakthrough of the but-1-ene with only slight increase with temperature. The steeper breakthrough with increasing temperature points to a faster equilibration. Selectivities in the adsorbed phase for this buta-1,3-diene/but-1-ene mixture are 7 to $4( \pm 1.5)$ at temperatures of 303 and $423 \mathrm{~K}$, respectively.

In order to fully assess the separation performance of the DD3R adsorbent, desorption from a column saturated with cis- or trans-but-2-ene was monitored as shown in Fig. 15. But-2enes only desorb at temperatures above $380 \mathrm{~K}$. Once this temperature is reached, full regeneration of the column is achieved.

\section{Discussion}

$\mathrm{HF}$ is usually the acid of choice for dissolving zeolites ${ }^{54,55}$ while $\mathrm{NaOH}$ is well known for selective leaching of $\mathrm{Si}^{56-58}$ Although
$\mathrm{KOH}$ is not commonly reported as a cleaning agent or for the dissolution of silica, it has been applied in the dissolution of silicate clays. ${ }^{56,57,59}$ The rate of silica dissolution decreases in the order of $\mathrm{K}^{+}>\mathrm{Na}^{+}>\mathrm{Li}^{+}{ }^{58-60}$ This is the result of the lower solvation diameter of $\mathrm{K}^{+}$in basic media, ${ }^{61}$ which enhances the adsorption of these cations on the $\mathrm{SiO}_{2}$ as compared to $\mathrm{Na}^{+}$(ref. 58) and results in a better cleaning effect. The destructive effectiveness of $\mathrm{KOH}$ is indicated by the use of Sigma-2 as seeds in a DDR synthesis mixture (Fig. 4), where otherwise only Sigma-2 would have been obtained. Although in the presence of $\mathrm{KOH}$ the synthesis $\mathrm{pH}$ is obviously higher than that of the standard synthesis, it is apparent that addition of $\mathrm{KOH}$ results in partial dissolution and corrosion of these Sigma-2 seeding crystals.

The experimental results strongly suggest that the addition of KF instead of the traditionally applied ethylenediamine has a positive influence on the formation of pure DD3R. When Method 1 is used, Sigma-2 is obtained in every case. Method 2 yielded pure DD3R crystals in only one out of the three syntheses. In every case also DD3R is formed in this method, but the ratio DD3R/Sigma 2 varies from synthesis to synthesis in such a way that the pattern of the DD3R is hardly visible. This behaviour was also observed by Gascon et al. ${ }^{13}$ and explained as the possible segregation of EDA from the water phase. In contrast, by using Method 3 (KF) high purity DD3R is always obtained.

Characterizing the as-synthesized DD3R crystals of Method 3, ${ }^{13} \mathrm{C}$ (MAS) NMR studies revealed that adamantylamine is not protonated, in line with previous studies. ${ }^{25}$ At the same time, ${ }^{19} \mathrm{~F}$ MAS NMR revealed the absence of fluoride species in the assynthesized as well as in the calcined DD3R crystals. This indicates that fluoride does not act as structure directing agent in the 
a)

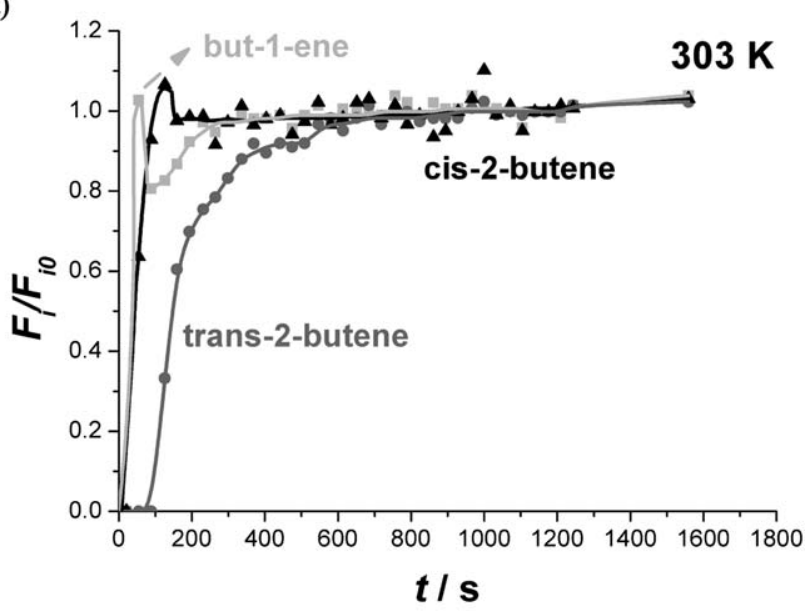

b)

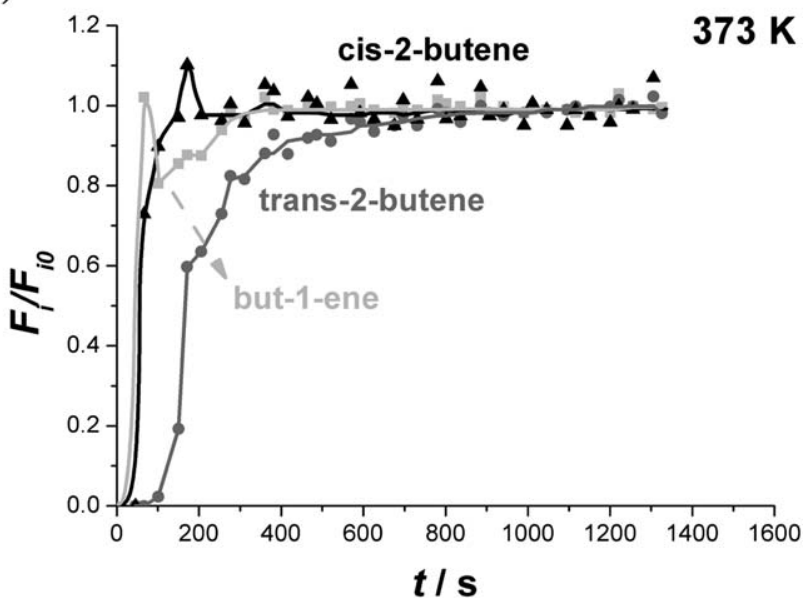

Fig. 13 Normalised molar flows as a function of time of but-1-ene, cisbut-2-ene and trans-but-2-ene in a ternary mixture $(1: 1: 1)$ at $120 \mathrm{kPa}$ : (a) $303 \mathrm{~K}$ and (b) $373 \mathrm{~K}$.

final stage of the zeolite synthesis, since they would probably have ended up in the small cages. Furthermore, ${ }^{29} \mathrm{Si}$ MAS NMR analysis revealed the absence of structural defects in the DD3R samples, also suggested by Fyfe et al. ${ }^{50}$ However, we cannot discard that the absence of $\mathrm{Si}-\mathrm{O}$ defects promotes the formation of the DD3R framework. ${ }^{34}$ Indeed, it has been suggested that $\mathrm{F}^{-}$ ions adsorb at the surface of particles under hydrothermal conditions and catalyze the formation of $\mathrm{Si}-\mathrm{O}-\mathrm{Si}$ bonds. ${ }^{34,39,62}$

Jon et al. ${ }^{63}$ showed that addition of $\mathrm{Na}$ cations to the synthesis medium increased the nucleation time in the case of all-silica BEA synthesis, where $\mathrm{NH}_{4} \mathrm{~F}$ is used as fluoride source. This was correlated to the earlier formation of $\mathrm{Na}^{+}$containing pentacoordinated silicon species rather than the $\mathrm{TEA}^{+}$containing pentacoordinated silicon species, which are instead needed for the zeolite beta formation. This pentacoordinated silicon species arises from hexafluorinated silicon, which is hydrolyzed to yield silica bridges and form TEAF species in basic medium. Formation of pentacoordinated species is mentioned by many other researchers as well. ${ }^{34,38,39}$ Although a similar mechanism could be proposed in the case of DD3R, it has to be considered that in this case the template is not cationic and therefore charge compensation is not needed. a)

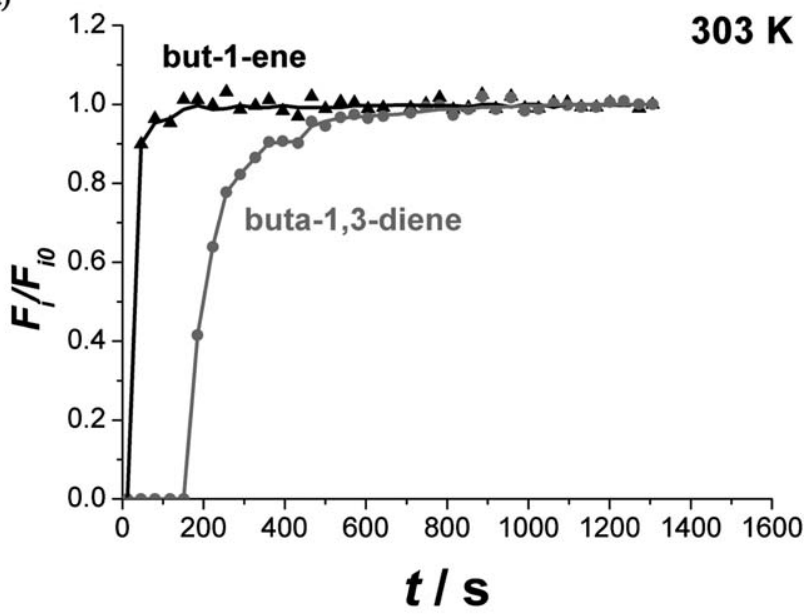

b)

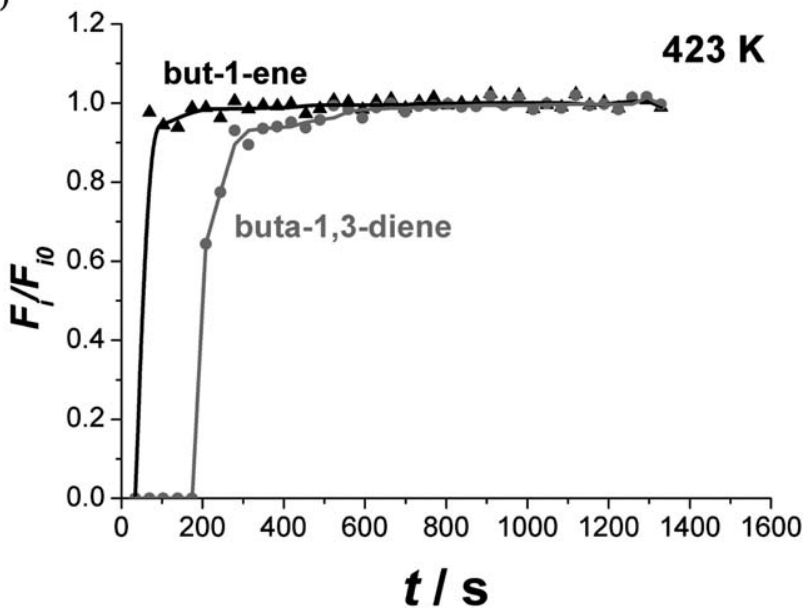

Fig. 14 Normalised molar flows as a function of time of buta-1,3-diene and but-1-ene mixture in a binary mixture of $(1: 1)$ at $120 \mathrm{kPa}$ : (a) $303 \mathrm{~K}$, (b) $373 \mathrm{~K}$ and (c) $423 \mathrm{~K}$.

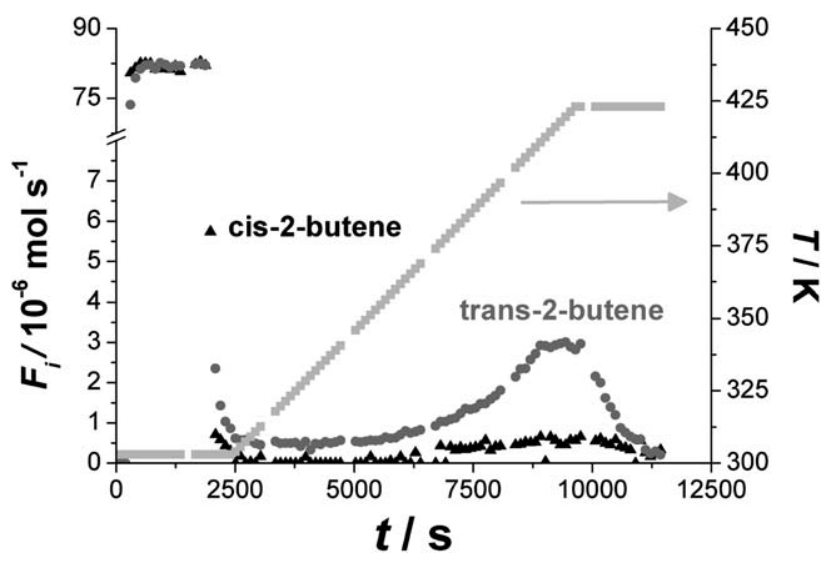

Fig. 15 Temperature programmed desorption in a helium flow of cis (black) and trans-but-2-ene (grey) from a saturated column (single component) at $120 \mathrm{kPa}$.

den Exter et al. $^{43}$ also studied the addition of less volatile amines for a better solubilisation of ADA. However, the use of bulkier amines with higher boiling points than ethylenediamine 
did not result in pure DD3R. Instead, rotation was introduced to overcome phase segregation of ADA from the solution phase. Apparently, KF is able to assist the solubilisation of ADA, ${ }^{64,65}$ even at the high synthesis temperatures.

These facts, together with the well known structure stabilization properties of $\mathrm{K}^{+}, 32,66$ bring us to the conclusion that the better reproducibility and high quality crystals obtained in the fluoride route are two-fold: KF suppresses the formation of lattice defects and helps solubilizing the ADA template.

Monitoring the crystallinity by XRD revealed also a phase transformation from Sigma-2 to DD3R. This fact can be due either to the dissolution of such crystals or to a decrease in their velocity of formation. SEM pictures revealed an intergrowth over the Sigma-2 crystals (Fig. 11) and even the growth of DD3R crystals over these intergrown particles, which clearly points to a solution mediated transformation. ${ }^{67,68}$ We speculate that the presence of KF in the synthesis solution has an important influence on this transformation. Burton et al. ${ }^{69}$ estimated the defect energy penalties of all-silica frameworks by making a correlation between the energetic penalty and defect concentration. Estimated defect energy penalties according to this correlation for SGT and DD3R phases are 7.2 and $5.8 \mathrm{~kJ} \mathrm{~mol}^{-1} \mathrm{SiO}_{2}$. High defect density of the SGT phase and the similarity of the ion radii for $\mathrm{O}^{2-}$ (1.4) and $\mathrm{F}^{-}$(1.36) allow us to suggest that highly active fluoride replaces oxygen ions at these defects and fill anion vacancies (healing effect) ${ }^{34,39}$ Formation of this fluorinated structure would lead to a fast consumption of the SGT phase. During the synthesis $\mathrm{KF} / \mathrm{SiO}_{2}$ ratio will increase in the synthesis solution due to formation of crystals, which also explains why these metastable phases are formed after certain synthesis time. This is further supported by the positive effect of an increase in the $\mathrm{KF} / \mathrm{SiO}_{2}$ ratio, which results in a faster dissolution of SGT crystals and hence a faster production of DDR (see Fig. 12).

TGA analysis in air of the as-synthesized and dried samples yielded a relative weight loss of $11 \%$, the theoretical weight loss for DD3R, ${ }^{25}$ indicating the purity of the product. The $80 \%$ yield of DD3R in the synthesis with the fluoride addition is also quite high, which is another important aspect of the synthesis method. The specific BET surface areas and pore volumes obtained are in a good agreement with the literature. ${ }^{25,70}$

As to the separation results, Fig. 13 demonstrates that DD3R is a powerful shape selective adsorbent for the separation of trans-but-2-ene from both cis-but-ene and but-1-ene. Although somehow similar selectivities have been claimed for other zeolites like Na/CaA (LTA), Erionite, AlPO-17 (ERI), all-silica chabazite (CHA) or ITQ-32 (IHW), ${ }^{24}$ this was all based on single component uptake data. The presented results are a prime example of an adsorbent for which this selectivity has been demonstrated for gas mixtures, apart from the reported liquid phase but-1-ene/cis-but-2-ene and but-1-ene/trans-but-2-ene separation on RUB-41 type zeolite. ${ }^{24}$ Moreover, not many examples exist in the literature of zeolitic adsorbents able to efficiently separate cis/trans isomers. ${ }^{71,72}$

The kinetic diameter of trans-but-2-ene and buta-1,3-diene $(0.431 \mathrm{~nm})$ is slightly smaller than the free cross diameter of the 8 -membered $(0.44 \times 0.36 \mathrm{~nm})$ ring allowing the trans isomer and buta-1,3-diene to enter the 19-hedron cavities. In contrast, the kinetic diameter of cis-but-2-ene is larger than the window size of the 8 -membered ring while the critical diameter of but-1-ene is comparable to the 8-ring aperture. The adsorption of cis-but-2-ene at both temperatures obtained was also recorded by Zhu et $_{\text {al. }}{ }^{22}$ and two possibilities were considered: adsorption on the external surface of the DD3R crystals or the possible presence of defects in the DD3R structure. Considering the high quality structure of the DD3R crystals, these results should be attributed to the adsorption on the external surfaces. Zhu et al., ${ }^{22}$ based on single component adsorption, reported trans-but-2-ene saturation loadings on DD3R of approximately $0.82 \mathrm{~mol} \mathrm{~kg}^{-1}$ (less than 1 molecule per 19-hedron cavity). In the dynamic competitive breakthrough experiments the obtained trans-but-2-ene loading is slightly lower, although the total loading on the crystal is higher, probably the consequence of cis-but-2-ene adsorption at the outer crystal surface.

In the case of binary adsorption experiments of but-1-ene and buta-1,3-diene, selective uptake of buta-1,3-diene is achieved. Increasing the temperature from $303 \mathrm{~K}$ to $423 \mathrm{~K}$ had almost no effect on the breakthrough times whereas a faster equilibration is achieved. Apart from the high purity of but-1-ene obtained in the breakthrough, the adsorption selectivities for buta-1,3-diene are fairly high, in the order of 4-7.

As mentioned earlier, in the current $\mathrm{C} 4$ isomers separation scheme, a combination of different methods is applied. Extractive distillation has been shown to discriminate between most of the isomers except for the but-1-ene/buta-1,3-diene couple. In the latter case, the solubility of both olefins is very similar in most organic solvents. The excellent separation performance shown by DD3R for this mixture might represent an attractive alternative to extractive distillation and finally pave the way for an economically feasible adsorptive separation of the but-1-ene and buta-1,3-diene mixture. The high temperature regeneration to remove the adsorbed components suggests that for the application of this separation the combination of temperature and pressure swing will be the best choice. In combination with the high selectivity for propene over propane the all-silica DD3R is among the most promising adsorbents for shape selective separations of small hydrocarbons.

\section{Conclusions}

In spite of the number of publications dealing with the synthesis of DD3R, reproducibility is still an issue. In this work we have shown that successful synthesis relies on two main pillars: the use of properly cleaned autoclave liners and the use of a proper DD3R synthesis composition. High DD3R purities are obtained when $\mathrm{KOH}$ is used as a cleaning agent of the PTFE autoclave liners, and when $\mathrm{KF}$ is added to the synthesis mixture. By means of ${ }^{13} \mathrm{C},{ }^{19} \mathrm{~F}$ and ${ }^{29} \mathrm{Si}$ (MAS) NMR it is shown that the crystals produced are defect free, and that fluoride addition induces neither a templating effect nor protonation of the template. The presence of KF in the synthesis medium enables better solubilisation of ADA, eliminating the need of autoclave rotation to induce mixing, and suppresses the formation of lattice defects.

DD3R is a powerful adsorbent for the separation of but-2-ene isomers. The performed breakthrough experiments reveal that the 8-ring apertures are accessible to trans-but-2-ene and buta1,3-diene, while they exclude but-1-ene and cis-but-2-ene molecules, as shown by ternary and binary adsorption profiles. This shape-selective adsorption is purely the result of differences in the kinetic diameters of the different adsorbates. 


\section{Acknowledgements}

Part of the research leading to these results has received funding from the European Community's Seventh Framework Programme through the Collaborative Project NEXT-GTL, under agreement no. 229183. Jorge Gascon gratefully acknowledges the Netherlands National Science Foundation (NWO-VENI) for financial support. We also acknowledge Dr Johan Groen, Dr Ger Koper and Dr Marcel den Exter for fruitful discussions.

\section{References}

$1 \mathrm{~W}$. Vermeiren and J. P. Gilson, Impact of zeolites on the petroleum and petrochemical industry, Top. Catal., 2009, 52, 1131-1161.

2 D. W. Breck, Zeolite Molecular Sieves: Structure, Chemistry and Use, 1974.

3 C. S. Cundy and P. A. Cox, The hydrothermal synthesis of zeolites: precursors, intermediates and reaction mechanism, Microporous Mesoporous Mater., 2005, 82, 1-78.

4 C. S. Cundy and P. A. Cox, The hydrothermal synthesis of zeolites: history and development from the earliest days to the present time, Chem. Rev., 2003, 103, 663-702.

5 L. Tosheva and V. P. Valtchev, Nanozeolites: synthesis, crystallization mechanism, and applications, Chem. Mater., 2005, 17, 2494-2513.

6 C. E. A. Kirschhock, S. P. B. Kremer, J. Vermant, G. Van Tendeloo, P. A. Jacobs and J. A. Martens, Design and synthesis of hierarchical materials from ordered zeolitic building units, Chem.-Eur. J., 2005, 11, 4306-4313.

7 H. Gies, B. Marler and U. Werthmann, Synthesis of porosils: crystalline nanoporous silicas with cage- and channel-like voids, Mol. Sieves, 1998, 1, 35-64.

8 A. Stewart, Zeolite Sigma-1, in United Kingdom Patent Application, 1988.

9 H. Gies, Studies on clatherasis VI: crystal structure of decadodecasil 3R the missing link between zeolites and clathrasils, Z. Kristallogr., 1986, 175, 93-104.

10 E. W. Valyocsik, D. H. Olson and P. G. Rodewald, A Crystalline Silicate and Method of its Synthesis, in European patent, 1986.

$11 \mathrm{~S}$. Ernst and J. Weitkamp, Hydrothermal synthesis of the zeolite ZSM-58 and template-free synthesis of the zeolite ZSM-5, Chem. Ing. Tech., 1991, 63, 748-750.

12 J. C. Jansen, Chapter 4 The Preparation of Molecular Sieves, in Studies in Surface Science and Catalysis, ed. E. M. F. H. van Bekkum and J. C. Jansen, Elsevier, 1991, pp. 77-136.

13 J. Gascon, W. Blom, A. van Miltenburg, A. Ferreira, R. Berger and F. Kapteijn, Accelerated synthesis of all-silica DD3R and its performance in the separation of propylene/propane mixtures, Microporous Mesoporous Mater., 2008, 115, 585-593.

14 Y. Kumita, J. Gascon, E. Stavitski, J. A. Moulijn and F. Kapteijn, Shape selective methanol to olefins over highly thermostable DDR catalysts, Appl. Catal., A, 2011, 391, 234-243.

$15 \mathrm{~J}$. van den Bergh, A. Tihaya and F. Kapteijn, High temperature permeation and separation characteristics of an all-silica DDR zeolite membrane, Microporous Mesoporous Mater., 2010, 132, 137 147.

16 J. van den Bergh, W. Zhu, F. Kapteijn, J. A. Moulijn, K. Yajima, K. Nakayama, T. Tomita and S. Yoshida, Separation of $\mathrm{CO}_{2}$ and $\mathrm{CH}_{4}$ by a DDR membrane, Res. Chem. Intermed., 2008, 34, 467-474.

17 J. Kuhn, J. M. Castillo-Sanchez, J. Gascon, S. Calero, D. Dubbeldam, T. J. H. Vlugt, F. Kapteijn and J. Gross, Adsorption and diffusion of water, methanol, and ethanol in allsilica DD3R: experiments and simulation, J. Phys. Chem. C, 2009, 113, 14290-14301.

18 J. van den Bergh, C. Gücüyener, J. Gascon and F. Kapteijn, Isobutane dehydrogenation in a DD3R zeolite membrane reactor, Chem. Eng. J., 2011, 166, 368-377.

19 J. Kuhn, K. Yajima, T. Tomita, J. Gross and F. Kapteijn, Dehydration performance of a hydrophobic DD3R zeolite membrane, J. Membr. Sci., 2008, 321, 344-349.
20 J. van den Bergh, W. Zhu, J. Gascon, J. A. Moulijn and F. Kapteijn, Separation and permeation characteristics of a DD3R zeolite membrane, J. Membr. Sci., 2008, 316, 35-45.

21 J. van den Bergh, W. Zhu, J. C. Groen, F. Kapteijn, J. A. Moulijn, K. Yajima, K. Nakayama, T. Tomita and S. Yoshida, Natural Gas Purification with a DDR Zeolite Membrane; Permeation Modelling with Maxwell-Stefan Equations, in Studies in Surface Science and Catalysis, 2007, pp. 1021-1027.

22 W. Zhu, F. Kapteijn, J. A. Moulijn and J. C. Jansen, Selective adsorption of unsaturated linear $\mathrm{C}_{4}$ molecules on the all-silica DD3R, Phys. Chem. Chem. Phys., 2000, 2, 1773-1779.

23 W. Zhu, F. Kapteijn, J. A. Moulijn, M. C. den Exter and J. C. Jansen, Shape selectivity in adsorption on the all-silica DD3R, Langmuir, 2000, 16, 3322-3329.

24 B. Tijsebaert, C. Varszegi, H. Gies, F. S. Xiao, X. Bao, T. Tatsumi, U. Müller and D. De Vos, Liquid phase separation of 1-butene from 2-butenes on all-silica zeolite RUB-41, Chem. Commun., 2008, $2480-2482$.

25 M. J. den Exter, J. C. Jansen, H. van Bekkum and A. Zikánova, Synthesis and characterization of the all-silica 8-ring Clathrasil DD3R comparison of adsorption properties with the hydrophilic zeolite A, Zeolites, 1997, 19, 353-358.

$26 \mathrm{~K}$. Yajima and K. Nakayama, Process for Producing DDR type Zeolite Membrane, United States patent and trademark office pregrant publication, 2009.

27 Q. L. Yang, S. L. Zhong and X. Lin, Synthesis of DDR-type zeolite in fluoride medium, Chin. J. Inorg. Chem., 2009, 25, 191-194.

$28 \mathrm{H}$. Kessler, Synthesis in the presence of fluoride high-silica zeolites, aluminophosphates and derived materials, Microporous Mesoporous Mater., 1998, 22, 517-518.

29 H. Kessler, J. Patarin, C. Schott-Darie, M. S. H. G. K. J. C. Jansen and J. Weitkamp, The Opportunities of the Fluoride Route in the Synthesis of Microporous Materials, in Studies in Surface Science and Catalysis, Elsevier, 1994, pp. 75-113.

30 P. Caullet, J. L. Paillaud, A. Simon-Masseron, M. Soulard and J. Patarin, The fluoride route: a strategy to crystalline porous materials, C. R. Chim., 2005, 8, 245-266.

$31 \mathrm{X}$. Liu, U. Ravon and A. Tuel, Evidence for $\mathrm{F}^{-} / \mathrm{SiO}^{-}$anion exchange in the framework of as-synthesized all-silica zeolites, Angew. Chem., Int. Ed., 2011, 1-5.

32 R. F. Lobo, S. I. Zones and M. E. Davis, Structure-direction in zeolite synthesis, J. Inclusion Phenom., 1995, 21, 47-78.

33 P. M. Piccione, S. Yang, A. Navrotsky and M. E. Davis, Thermodynamics of pure-silica molecular sieve synthesis, J. Phys. Chem. B, 2002, 106, 3629-3638.

34 L. A. Villaescusa, P. S. Wheatley, I. Bull, P. Lightfoot and R. E. Morris, The Location and ordering of fluoride ions in pure silica zeolites with framework types IFR and STF; implications for the mechanism of zeolite synthesis in fluoride media, J. Am. Chem. Soc., 2001, 123, 8797-8805.

35 C. M. Zicovich-Wilson, F. Gàndara, A. Monge and M. A. Camblor, In situ transformation of TON silica zeolite into the less dense ITW: structure-direction overcoming framework instability in the synthesis of $\mathrm{SiO}_{2}$ zeolites, J. Am. Chem. Soc., 2010, 132, 34613471.

36 M. A. Camblor, L. A. Villaescusa and M. J. Diaz-Cabanas, Synthesis of all-silica and high-silica molecular sieves in fluoride media, Top. Catal., 1999, 9, 59-76.

37 O. Larlus and V. P. Valtchev, Control of the morphology of all-silica BEA-type zeolite synthesized in basic media, Chem. Mater., 2005, 17, 881-886.

38 H. Koller, A. Wölker, H. Eckert, C. Panz and P. Behrens, FiveCoordinate silicon in zeolites: probing $\mathrm{SiO}_{4} / 2 \mathrm{~F}^{-}$sites in Nonasil and ZSM-5 with ${ }^{29} \mathrm{Si}$ solid-state NMR spectroscopy, Angew. Chem., Int. Ed. Engl., 1997, 36, 2823-2825.

39 L. A. Villaescusa, P. A. Barrett and M. A. Camblor, Calcination of octadecasil: fluoride removal and symmetry of the pure $\mathrm{SiO}_{2}$ host, Chem. Mater., 1998, 10, 3966-3973.

40 X. Yang, D. Albrecht and J. Caro, Solvothermal synthesis of germanosilicate-sodalite and silica-sodalite: effects of water, germanium and fluoride, Microporous Mesoporous Mater., 2007, 100, 95-102.

41 S. I. Zones, R. J. Darton, R. Morris and S.-J. Hwang, Studies on the role of fluoride ion $v s$. reaction concentration in zeolite synthesis, $J$. Phys. Chem. B, 2005, 109, 652-661. 
42 H. Gies, B. Marler, U. Werthmann, Synthesis of Porosils: Crystalline Nanoporous Silicas with Cage- and Channel-like Voids, in Molecular Sieves: Science and Technology, 1998, pp. 35-64.

43 M. J. den Exter, in Exploratory Study of the Synthesis and Properties of 6-, 8- and 10-Ring Tectosilicates and their Potential Application in Zeolite Membranes, TU Delft, Delft, 1996, pp. 1-223.

44 T. Kakigi, A. Oshima, N. Miyoshi, K. Murata, K. Fujii, N. Mitani, T. Urakawa, Y. Sato, A. Matsuura, J. Li, T. Miura, E. Katoh and M. Washio, Study on chemical structures of poly (tetrafluoroethylene-co-perfluoroalkylvinylether) by soft-EB irradiation in solid and molten state, Nucl. Instrum. Methods Phys. Res., Sect. B, 2007, 265, 118-124.

45 A. van Miltenburg, J. Gascon, W. Zhu, F. Kapteijn and J. A. Moulijn, Propylene/propane mixture adsorption on faujasite sorbents, Adsorption, 2008, 14, 309-321.

46 Database of zeolite structures, http://www.iza-structure.org/ databases/, accessed January 2011.

47 H. Kabashima, H. Tsuji, S. Nakata, Y. Tanaka and H. Hattori, Activity for base-catalyzed reactions and characterization of alumina-supported KF catalysts, Appl. Catal., A, 2000, 194-195, $227-240$.

48 J. M. Clacens, D. Genuit, L. Delmotte, A. Garcia-Ruiz, G. Bergeret, R. Montiel, J. Lopez and F. Figueras, Effect of the support on the basic and catalytic properties of KF, J. Catal., 2004, 221, 483-490.

49 B. Fèron, J. L. Guth and N. Mimouni-Erddalane, Influence of the presence of $\mathrm{NaF}$ on the crystallization of zeolite A (LTA): first evidence for the existence of fluorosodalite, the missing end-member of the halosodalite series, Zeolites, 1994, 14, 177-181.

50 C. A. Fyfe, H. Gies, Y. Feng and H. Grondey, Two-dimensional ${ }^{29} \mathrm{Si}$ MAS n.m.r. investigation of the three-dimensional structure of zeolite DD3R, Zeolites, 1990, 10, 278-282.

51 P. C. M. M. Magusin, V. E. Zorin, A. Aerts, C. J. Y. Houssin, A. L. Yakovlev, C. E. A. Kirschhock, J. A. Martens and R. A. Van Santen, Template-aluminosilicate structures at the early stages of zeolite ZSM-5 formation. A combined preparative, solid-state NMR, and computational study, J. Phys. Chem. B, 2005, 109, 22767-22774.

52 A. Çullfaz and L. B. Sand, Mechanism of nucleation and crystallization of zeolites from gels, Adv. Chem., 1973, 121, 140151.

53 L. B. Sand, Zeolite Synthesis and Crystallization, Proc. 5th Int. Conf./ Zeolites, 1980, pp. 1-9.

54 F. J. Langmyhr and P. E. Paus, The analysis of inorganic siliceous materials by atomic absorption spectrophotometry and the hydrofluoric acid decomposition technique: Part I. The analysis of silicate rocks, Anal. Chim. Acta, 1968, 43, 397-408.

55 F. J. Langmyhr and S. Sveen, Decomposability in hydrofluoric acid of the main and some minor and trace minerals of silicate rocks, Anal. Chim. Acta, 1965, 32, 1-7.

56 J. C. Groen, G. M. Hamminga, J. A. Moulijn and J. Perez-Ramirez, In situ monitoring of desilication of MFI-type zeolites in alkaline medium, Phys. Chem. Chem. Phys., 2007, 9, 4822-4830.
57 J. C. Groen, J. A. Moulijn and J. Perez-Ramirez, Desilication: on the controlled generation of mesoporosity in MFI zeolites, J. Mater. Chem., 2006, 16, 2121-2131.

58 J. C. Groen, J. A. Moulijn and J. Perez-Ramirez, Alkaline posttreatment of MFI zeolites. From accelerated screening to scaleup, Ind. Eng. Chem. Res., 2007, 46, 4193-4201.

59 M. Pentrak, J. Madejova and P. Komadel, Acid and alkali treatment of kaolins, Clay Miner., 2009, 44, 511-523.

60 A. C. Pierre, Introduction to Sol-Gel Processing, Kluwer Academic Publishers, Boston, 1998.

61 L. Degrève, S. M. Vechi and C. Q. Junior, The hydration structure of the $\mathrm{Na}^{+}$and $\mathrm{K}^{+}$ions and the selectivity of their ionic channels, Biochim. Biophys. Acta, Bioenerg., 1996, 1274, 149-156.

62 L. M. Lopato, A. V. Shevchenko, V. P. Red'ko and V. V. Pasichnyi, Theory, production technology, and properties of powders and fibers: features of solid solution formation with a fluorite type structure in the system $\mathrm{ZrO}_{2}-\mathrm{HfO}_{2}-\mathrm{Y}_{2} \mathrm{O}_{3}$ with different synthesis methods, Powder Metall. Met. Ceram., 2006, 45, 1-7.

63 H. Jon, Y. Oumi, K. Itabashi and T. Sano, Role of ammonium fluoride in crystallization process of beta zeolite, J. Cryst. Growth, 2007, 307, 177-184.

64 G. J. d. A. A. Soler-Illia, C. Sanchez, B. Lebeau and J. Patarin, Chemical strategies to design textured materials: from microporous and mesoporous oxides to nanonetworks and hierarchical structures, Chem. Rev., 2002, 102, 4093-4138.

$65 \mathrm{~J}$.-A. Ke and I. Wang, Elucidation of the role of potassium fluoride in the chemical and physical nature of ZSM-5 zeolite, Mater. Chem. Phys., 2001, 68, 157-165.

$66 \mathrm{~K}$. Byrappa and M. Yoshimura, in Handbook of Hydrothermal Technology - a Technology for Crystal Growth and Materials Processing, William Andrew Publishing/Noyes, 2001, pp. 315-414.

67 B. Subotic and L. Sekovanic, Transformation of zeolite A into hydroxysodalite: II. Growth kinetics of hydroxysodalite microcrystals, J. Cryst. Growth, 1986, 75, 561-572.

68 B. Subotic, D. Skrtic, I. Smit and L. Sekovanic, Transformation of zeolite A into hydroxysodalite: I. An approach to the mechanism of transformation and its experimental evaluation, J. Cryst. Growth, 1980, 50, 498-508.

69 A. W. Burton, G. S. Lee and S. I. Zones, Phase selectivity in the syntheses of cage-based zeolite structures: an investigation of thermodynamic interactions between zeolite hosts and structure directing agents by molecular modeling, Microporous Mesoporous Mater., 2006, 90, 129-144.

70 S. Himeno, T. Tomita, K. Suzuki and S. Yoshida, Characterization and selectivity for methane and carbon dioxide adsorption on the all-silica DD3R zeolite, Microporous Mesoporous Mater., 2007, 98, $62-69$.

71 G.-H. Hsiue and J.-S. Yang, $\mathrm{Ag}^{+}$contained complex membrane for the separation of $\mathrm{C}_{4}$ olefin/paraffin mixture, J. Polym. Res., 1994, 1, $35-41$.

72 H. Faghihian and M. Pirouzi, Cis/trans-but-2-ene adsorption on natural and modified clinoptilolite, Clay Miner., 2009, 44, 405-409. 